\title{
Italique
}

Poésie italienne de la Renaissance

XXI| 2018

Poesia e Religione

\section{Poesia biblica a Firenze nell'età del Savonarola}

\section{Erminia Ardissino}

\section{OpenEdition}

\section{Journals}

Edizione digitale

URL: http://journals.openedition.org/italique/605

DOI: 10.4000/italique.605

ISSN: 1663-4438

\section{Editore}

Librairie Droz

\section{Edizione cartacea}

Data di pubblicazione: 1 novembre 2018

Paginazione: 1-28

ISBN: 978-2-600-05924-4

ISSN: 1423-3983

Notizia bibliografica digitale

Erminia Ardissino, «Poesia biblica a Firenze nell'età del Savonarola», Italique [Online], XXI | 2018, online dal 01 novembre 2020, consultato il 25 janvier 2021. URL: http://journals.openedition.org/italique/605 ; DOI: https://doi.org/10.4000/italique.605 
Erminia Ardissino

P O E S I A B I B L I C A

A FIRENZE NELL'ET À

D E L S A V O N A R L A 



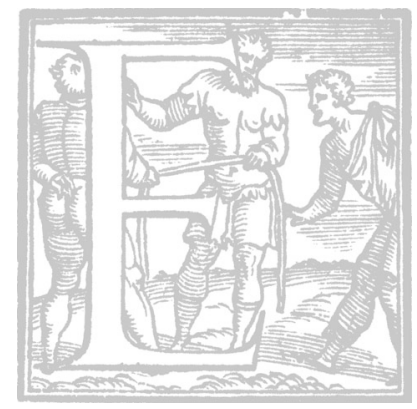

ben noto che, nel delineare la figura del suo principe ideale, Machiavelli nel capitolo sesto del suo trattato ne confronta le caratteristiche con Mosé, oltre che con altri personaggi storici o mitologici come Ciro, Romolo, Teseo, e che nell'esortazione finale al dedicatario, Lorenzo de' Medici, invitandolo a divenire guida di un riscatto dell'Italia, al presente "più stiava che li Ebreì, di nuovo richiama la figura di Mosè, come modello di guida per sollecitare «la virtù d'uno spirito italiano». 'Meno noto è che il Segretario fiorentino fu anche (seppur per una minima porzione di testo) traduttore dei Salmi, come appare in un'esortazione alla penitenza, scritta probabilmente negli ultimi anni di vita per una confraternita fiorentina, in cui inserisce citazioni dal cinquantesimo salmo, il Miserere, in latino e in volgare, per accompagnare osservazioni sulla misericordia divina. Se all'avvio Machiavelli cita il versetto introduttivo in latino: "De profundis clamavi ad te, Domine, Domine, exaudi vocem meam», poi invita $i$ fedeli a ripetere le parole di David, il "Profeta», traducendo il seguito: "O Signore, io constituto nel profondo del peccato, ho con voce umile e piena di lacrime, chiamato a te, o Signore, misericordia; e ti priego, e tu sia contento, per la tua infinita bontà concedermelà. ${ }^{2}$

La cultura biblica di Machiavelli non sorprende, se si considerano i suoi interessi per l'antichità, della cui politica alcuni libri biblici trattano. Ma egli aveva anche conosciuto da giovane l'intensa spinta religiosa che Firenze aveva vissuto sotto il reggimento di Savonarola, che tra l'altro si proponeva come guida del popolo fiorentino verso la renovatio che avrebbe dovuto trasformare la città in una nuova guida della cristianità, abbandonando, come gli Ebrei, la schiavitù dei beni terreni. Ma ancor meno sorprende se si considera la lunga tradizione di letture, riflessioni, rielaborazioni e cultura bibliche che caratterizzarono la Firenze umanistica e mediceo-laurenziana. Se da una parte la riscoperta e valorizzazione dell'antichità con lo splendore della sua arte autorizzavano a proporla come la nuova Atene, dall'altra correva parallelo un altrettanto intenso interesse per la Bibbia, eredità non certo solo dell'ondata di entusiasmo per i Padri portata dal Concilio del $1439,{ }^{3}$ ma soprattutto dell'intensa predicazione dei suoi santi o uomini di Chiesa, come il 
santo vescovo Antonino Pierozzi, ${ }^{4}$ che esortava persino le donne alla lettura della Bibbia.' Nel suo trattato Opera a ben vivere il santo vescovo raccomanda di abbinare alla preghiera la lettura e in particolare di meditare ogni giorno sulla passione di Cristo, dando delle indicazioni dettagliate e focalizzate sui dolori della passione. L'opera del Pierozzi, dedicata dapprima a Diodora Tornabuoni e poi alla sorella Lucrezia, moglie di Piero de' Medici e madre di Lorenzo, fu evidentemente feconda se nella sua maturità Lucrezia compose ben cinque poemetti biblici. $^{6}$

L'opposizione Cristianesimo-Umanesimo paganeggiante, avanzata pure a Firenze dal domenicano Giovanni Dominici nel suo Lucula noctis, non aveva avuto presa sulla città, ${ }^{7}$ anzi si può con più sicurezza affermare che Umanesimo e cultura biblica camminarono unitamente e contribuirono insieme a rendere splendida la città di Firenze, nuova Atene, come volevano Salutati e Poliziano, ma anche nuova Gerusalemme, come proponeva Savonarola. ${ }^{8}$ Basti pensare che Marsilio Ficino, promotore di quel «ritorno di Platone», attuato con le traduzioni e i commenti dei dialoghi platonici e delle opere dei seguaci Plotino e Proclo, oltre che sacerdote, traduttore e commentatore dei mistici scritti di Dionigi Areopagita, era anche commentatore delle epistole di san Paolo e volgarizzatore dei Salmi (o meglio, di un compendio di invocazioni tratte dai salmi, destinato alla famiglia Medici).

Firenze aveva anche conosciuto il primo tentativo «moderno» di ricostruzione del testo biblico: già negli anni '4o del XV secolo Giannozzo Manetti aveva iniziato una nuova tradurione della Bibbia dall'ebraico. ${ }^{\text {IO }}$ Sebbene l'impresa non fosse poi stata compiuta e il suo autore fosse passato a Roma, aveva comunque lasciato a Firenze un'eredità che approdò a fine secolo agli studi biblici di Giovanni Pico della Mirandola, $i$ cui scritti maggiori sono commenti alla Bibbia $o$ dalla Bibbia ispirati. ${ }^{\text {I }}$ Il lavoro di Manetti, come quello di umanisti come Niccolò Niccoli e Ambrogio Traversari, significò anche un ritorno alle Sacre Scritture e l'avvio di una filologia biblica, che ebbe poi nel secolo successivo, nelle imprese bibliche di Sante Pagnini e nella scuola ebraica di San Marco, degni successori. ${ }^{2}$

Se cosi vivo fu l'interesse di filologi e nomini di Chiesa a Firenze per le Sacre Scritture, non meno avvenne sul versante poetico: a metà Quattrocento Domenico di Giovanni da Corella compose un poema in distici elegiaci, Theotocon, sulla Madre di Dio, imitato poi dai poemi latini 
di Ugolino Verino, autore di una trasposizione in esametri della Bibbia, il Poema sacrum Veteris et Novi Testamenti. ${ }^{{ }^{3}}$ Persino Poliziano compose un Pater Noster in esametri dattilici e due inni alla Vergine. ${ }^{\mathrm{I} 4}$

Anche in ambito volgare la letteratura attesta che la Bibbia doveva essere al centro della vita culturale fiorentina in età umanistica, basti pensare a opere come La città di vita di Matteo Palmieri o il commento alla Commedia di Cristoforo Landino, che alla Bibbia si ispirano. ${ }^{15}$ Ma molte altre prove bibliche poetiche in volgare si possono enumerare nella Firenze del Quattrocento, ed è proprio di questa produzione che intendiamo qui occuparci, limitandoci all'ultimo quarto di secolo. Si può affermare che la predicazione biblica di Savonarola non crescesse in un campo privo di corrispondente interesse, perché la Bibbia apparteneva alla cultura di Firenze alla fine del Quattrocento forse più che in altri centri culturali italiani. La storiografia ha prestato davvero poca attenzione a questa produzione fiorentina biblica in volgare, che è stata invece ricca e abbondante, interessata soprattutto al Nuovo Testamento, in particolare alle figure di Gesù e di Maria. ${ }^{16}$ Molti di questi testi si devono ancora leggere in antiche stampe, incunaboli e cinquecentine, o addirittura in manoscritti. Spesso si tratta di una produzione laudistica legata alle confraternite, ma anche di poemi o di riscritture versificate di episodi evangelici e di molte sacre rappresentazioni. La stessa corte medicea fu coinvolta in questa rielaborazione della Bibbia: abbiamo già detto di Lucrezia, ed è noto che il figlio Lorenzo compose laudi e una Sacra rappresentazione di Giovanni e Paolo. ${ }^{17}$ A completare il quadro è pure rilevante l'arte biblica patrocinata dai Medici, che usò le figure bibliche per messaggi civili, come avvenne per la Giuditta di Donatello, cui sembra fosse apposta una scritta, voluta da Piero dei Medici, che esaltava l'eroina come emblema di libertà. ${ }^{18}$

La produzione poetica biblica fiorentina circolata a stampa nell'ultimo quarto del Quattrocento, di origine per lo più laica, era anche diretta ai laici, in funzione educativa, devozionale, di intrattenimento. Si tratta di testi popolari di facile accesso ad uso di persone di non altissima cultura: illetterati in quanto non capaci di intendere il latino, anche se alfabetizzati. A questi lettori si agginngevano tutti coloro che potevano fruirne tramite l'ascolto di recitazioni e letture ad alta voce, pratica molto frequente soprattutto in ambito devozionale. ${ }^{\text {'9 }}$ 
Pur volendo tralasciare l'esperienza molto significativa dell'uso biblico da parte delle tre corone fiorentine, Dante, Petrarca e Boccaccio, non si può evitare di imbattersi subito nel nome del primo dei tre: Dante. Appena si scorre la lista delle pubblicazioni bibliche quattrocentesche in volgare, ${ }^{20}$ si incontra ben presto il nome di Dante, ritenuto autore di una fortunata raccolta di salmi penitenziali in terza rima, Incomincia gli setti psalmi penitentiali uulgarmente composti, edita per la prima volta a Firenze da Johannis Petri nel I47I, ${ }^{2 \mathrm{I}}$ lo stesso anno delle due prime stampe di traduzioni dell'intera Bibbia uscite a Venezia in agosto e in ottobre, agli albori della «rivoluzione inavvertita). Si tratta di un piccolo incunabolo in- $4^{\circ}$ di sole I4 carte, che raccoglie un testo tramandato da numerosi manoscritti e poi riedito numerose volte. ${ }^{22}$ L'attribuzione a Dante, riconosciutagli da un autorevole studioso come Francesco Saverio Quadrio nel Settecento, è tuttavia assolutamente non convincente, perché lo stile dimesso si dimostra assai lontano dal poema e dalle rime di Dante, senza contare che una traduzione dei salmi sarebbe incongrua per chi si era decisamente espresso contro la traduzione del Salterio, cosi come di ogni «cosa per legame musaico armonizzata». In Convivio I VII I4-I6 Dante infatti attribuisce proprio al passaggio dall'ebraico al greco e dal greco al latino la mancanza di «dolcezza di musica e d'armonia» del salterio in uso. Inoltre, le molte citazioni dei salmi nella Commedia sono sempre in latino, mai tradotte. La scelta metrica (la terzina) ba certo facilitato l'attribuzione e persino le modalità traduttorie possono trarre in inganno, in quanto anche qui, come per il Pater noster dei superbi di Purgatorio XI I-24, il solo testo biblico davvero tradotto da Dante, viene assegnato a ogni versetto biblico una terzina che ne espande il contenuto. Come per l'oratio dominica recitata dai superbi, nei Setti psalmi penitentiali si inseriscono nel tessuto originale aggiunte attualizzanti; la procedura ha però come esito l'impiego di molte dittologie sinonimiche o perifrasi o comunque figure di amplificatio che servono per colmare $i$ vuoti. ${ }^{23}$ Talvolta appaiono parole o immagini derivate dall'opera di Dante, ma possono essere naturalmente rielaborazioni indipendenti.

I salmi, soprattutto quelli penitenziali, erano testi di largo uso non solo per la liturgia, ma anche per la preghiera individuale: ciò spiega la diffusione di queste terzine di uno pseudo-Dante, che ebbero ben dieci edizioni nel Quattrocento a Firenze e altrove (Bologna, Modena, 
Vicenza e soprattutto Venezia). Presto Firenze darà vita a nuove e rinnovate traduzioni dei salmi da parte di umanisti come Girolamo Benivieni e Luigi Alamanni, ma ambedue cadono ormai nel secolo successivo. ${ }^{24}$

A Dante nel Quattrocento è attribuita anche un'altra composizione d'impronta biblica, che ebbe l'onore della stampa: Il Credo, uscito dapprima a Roma nel i47s presso Johann Shurener, ebbe poi ben nove edizioni, per lo più fiorentine. ${ }^{25}$ L'incunabolo di 6 carte in-4 $4^{\circ}$ contiene terzine attribuite a Dante in occasione di un'accusa di eresia. Questo Credo volgare venne ristampato anche in successive edizioni del poema ed ebbe grande fortuna. Prima ancora che la Commedia apparisse a stampa (Venezia, Vendelinus de Spira, I477) questi testi (salmi e Credo) circolarono largamente attribuendo al sommo poeta opere che di suo ebbero solo il modello della terzina.

A questi «volgarizzamenti» pseudo-danteschi, prodotti nel Trecento ma ampiamente diffusi a Firenze con la stampa, si accompagnarono altri generi biblici, di impronta prettamente fiorentina, come $i$ cantari e le sacre rappresentazioni, ambedue generi molto popolari nella Firenze quattrocentesca. Tralasciamo qui l'abbondantissima produzione di sacre rappresentazioni, pure composte in poesia, perché è l'aspetto piu studiato della riscrittura biblica in volgare a Firenze all'epoca. ${ }^{26}$ Preferiamo focalizzare l'attenzione sulla produzione poetica dell'età savonaroliana, che costituisce una voce assai interessante nel panorama della poesia fiorentina che segue il «secolo senza poesia».

Anzitutto consideriamo i cantari, che, si sa, sono un genere orale, la cui tradizione scritta è piuttosto labile. Tra $i$ cantari che si recitavano in piazza san Martino si annoverano anche storie bibliche. ${ }^{27}$ Il più celebre e ammirato tra $i$ canterini, Antonio di Guido, mise in ottave una «sposizione del Testamento», nove stanze composte «a preghiera d'un serviziale di S. Maria Nuovas;; ${ }^{28}$ a Niccolò Cieco, che recitò molte storie sacre in volgare, è attribuita una storia di Susanna. ${ }^{29}$ Il genere ebbe una certa influenza sui poemi della Tornabuoni in ottave, infatti La Istoria di Iudith $e$ La vita di sancto Johanne Baptista si avvicinano sia nella forma sia nel modo di procedere al genere canterino, tanto che per lo più l'autrice si rivolge al suo pubblico come ascoltatori e non lettori, come appare evidente in alcune espressioni: «sta, auditor, con l'intellecto desto» (Baptista str. I33), «udite come» (str. 20) e «come voi udite» (str. 25, identico a un'espressione in Iudith «come voi udite», 
Iudith str. 64). Dagli appelli al lettore si ricava quindi che il genere in cui si collocano $i$ due poemi in ottave della Tornabuoni è inteso per la recitazione, mentre quelli in terzine sono considerati testi più meditativi e impegnativi, quindi per la lettura.

Il primo cantare biblico andato in stampa a Firenze nel I482 è però di origine senese La passione di Gesù Cristo di Nicolò Cicerchia. Dell'autore, seguace di santa Caterina, si sa solo che l'accompagnò ad Avignone nella sua missione presso il papa. I suoi due cantari di argomento evangelico, La passione di Gesù Cristo $e$ La resurrezione di Gesù, furono ripetutamente editi nel secolo degli incunaboli e si presentano come opere di meditazione e devozione ad uso di confraternite. ${ }^{30}$ Firenze se ne approprio, mandandoli a stampa e arrivando ad attribuirli ad autori fiorentini, al Boccaccio e a Bernardo Pulci. A nome di quest'ultimo l'opera del Cicerchia fu spesso edita, con piccole varianti, accompagnata al ternario su Maria Maddalena Il pianto della Maddalena, effettivamente del Pulci.

Bernardo Pulci fu anche autore di una Passione e di un capitolo in terzine sullo stesso tema: La passione del nostro Signore Iesù Christo. ${ }^{31}$ Si tratta di un poema in 207 ottave, composto, secondo la dedica a suor Annalena Tadini delle Murate di Firenze, per stimolare nei cristiani la partecipazione alle vicende della passione. Cosi vi si legge:

Et però, dilectissima mia, quando leggerai questi versi, fa che tu presti qualche lachryma insieme con quella isconsolata madre a piè del sanctissimo legno inginocchiata et dello suo giusto sangue dipinta. ${ }^{32}$

L'attacco del poema è dantesco, e anche più avanti si trovano prestiti dall'opera di Dante:

Tutti voi che passate per via attendete et vedete il mio dolore se nessuno è che al mondo maggior sia per Ieremia ti chiama il tuo Signore. Et così ti risveglia oggi Maria a pianger seco con pietoso core, mostrando in croce il suo figliuol che langue et versa per te ingrato il giusto sangue. ${ }^{33}$ 
Il poema è una fedele narrazione della passione secondo i vangeli sinottici (Mt 26 e 27; Mc I4 e I5; Lc 22, 23 e 24; Gv I8 e I9). L'atto finale della redenzione è letto sulla base delle figure del Vecchio Testamento che prefigurano Cristo, molti sono infatti i riferimenti al testo veterotestamentario. Il poema ha una sua originalità, poiché sono inseriti molti dialoghi, soprattutto fra Gesù e Maria, o anche monologhi, come il commento di Maria ai piedi della croce, che consentono di dar voce ai sentimenti, creando una forte partecipazione emotiva. Molto frequentemente sono inserite parole latine, specie nei discorsi di Gesù, quasi ilpoeta volesse conservare la fonte come da tradizione. Ad esempio nell' istituzione dell'eucarestia si legge «accipite et comedite, ancor disse / questo è il mio corpo» (str. 30); nell'episodio del Getsemani: «Padre, s'egli èpossibil, dal figliuolo / questo calice dur fa che sia tolto / et niente dimeno non sicut volo» (str. 37), ai discepoli dice: «Non potuistis mecum vigilare» (str. 38) e a Giuda: «amice, ad quid venistiv;; al ladrone in croce: «amen, hodie mecum in ciel sarai» (str. I09). Non è solo Gesù «che 'parla latino'», ma anche il popolo che a Pilato replica: «nos habemus in lege» (str. 63) e Pilato: «non licet quenquam interficere a noi» (str. $7 I$ ) e ancora "Pilato disse lor quod scripsi scripsi» (str. 95). La citazione è presente anche nella narrazione: «et super vestem meam miserunt sorte» (str. 94), «tenebre facte sunt infino a nona» (str. I29), che dimostra come queste frasi fossero intese e di dominio comune, anzi, proprio la fedeltà a forme divenute patrimonio comune sembrano dovute le citazioni.

La figura principale è sicuramente Maria, sia per il suo ruolo sia per le parole che le sono affidate. Con il suo pianto l'addolorata fa eco ai fatti, dando spessore emotivo alla narrazione. Nei discorsi di Maria sono riprese anche le vicende della vita di Gesù non pertinenti alla passione: l'infanzia, la predicazione, i miracoli, non posti in ordine cronologico, ma inseriti appropriatamente nel contesto della vicenda. Per esempio $i$ miracoli sono ricordati nel momento in cui $i$ Giudei chiedono la morte di Gesù, l'infanzia ritorna nelle parole di Maria al momento della deposizione. Il pianto di Maria a volte è un grido straziante, come ai piedi della croce: "Oimé, figliuol mio, chi mi t’ha tolto, / che fusti già di mia vita sostegno? / oimé, chi t'ha scurato il sancto volto / e sparso il sangue prezioso e degno?» (str. 105). A volte è un umano, intimo, lamento: "Ove ti lascio, mio dolce figlinolo, / dilecto padre, o mio celeste sposo, / come viver potrò sanza te solo? / dove riman nel monumento ascoso? / io non posso portar si greve duolo» (str. I83). 
Frequenti sono anche gli appelli rivolti non tanto al lettore, quanto al devoto, al popolo cristiano, all'anima, per invitare alla compunzione, a una migliore coscienza di quanto sia costata la redenzione. Vediamo per esempio le due ottave che seguono la crocifissione (str. I58-I59):

Raguarda il tuo Signor, popol cristiano, et di pianger con meco un po' ti piaccia; vedi che versa il giusto sangue umano co' piè conficti et con le sancte braccia, essendo tu da Dio facto lontano per suscitarti infino al cor si straccia, sacrificato come lui dispone, come pecora va all'uccisione.

Quanto più penso a tua pietà infinita tanto di dolce amor triema la mente, ché per me, creatura vil finita, a morte corre, ingrato et sconoscente. Anima, che nel mondo se' smarrita, raguarda in croce il tuo signor clemente, che col suo sangue le tua colpe appaga et ti mostra l'una et l'altra piaga.

La chiusura è formata da una serie di ottave di preghiera, per il poeta che «piangendo scrive la tua passione», per $i$ cristiani pellegrini «involti in questa valle obscura», per le Murate, "di Iesù poste a seguitar la vià. Nell'insieme lasciano l'impressione di aver ascoltato (non vi sono cenni alla lettura) un poema-preghiera, che ha comportato un atto di devozione partecipato.

Sulla Passione il Pulci compone anche un capitolo di ottanta terzine, che segue dal punto di vista narrativo e lirico il modello della passione in ottave, anche se con una concentrazione espressiva che lo rende piu atto alla lettura che alla recitazione. Anzitutto si apre con sette terzine di considerazione del sacrificio di Cristo, atto redentivo per l'umanità. Ogni episodio è riassunto in una terzina. Anche qui $i$ riferimenti veterotestamentari spiegano gli eventi. A volte si avvertono espressioni simili al poema in ottave, ad esempio nel pianto si legge (str. 69): 
Omé l'anima tua, omé il tuo scudo, padre, sposo dilecto, et figliuol pio a torto e in croce lacerato et nudo. ${ }^{34}$

Si evidenzia una chiara ripresa petrarchesca: "per la pietà del tuo signore immenso» (terzina 64), da Rerum vulgarium fragmenta 3. Il Lamento della Maddalena è ancora più breve, 45 terżine intese a «cantar le laude» della seguace di Cristo, identificata sia con la peccatrice sia con una delle sorelle di Lazaro, come compare già nel poema. Il ternario ripercorre nella prima parte gli episodi evangelici relativi alle due donne, come se si trattasse di un'unica protagonista, portandola ad esempio di chi deve lasciare il peccato e ritrovare la via del cielo. Nella seconda parte si racconta come la donna abbia seguito Cristo sul Calvario, poi alla tomba, ritornando il giorno seguente, quindi come sia stata eremita «nella spelonca orribile e diserta» di Marsiglia dove stette «trent'anni ascosa». Infine le ultime sei terzine sono una preghiera alla santa affinché supplichi Gesù per "ch'io sia degno del ciel qual tu possiedi / viver al fin di questo carcer tetro» (str. 45). ${ }^{35}$

Molta attenzione a Firenze fu dedicata al Battista, figura ovviamente di rilievo in quanto patrono della città. Si registra un anonimo poema in ottave, che ebbe più edizioni anche con diversi titoli: La vita et morte di Sancto Joanne Baptista. ${ }^{36}$ Si tratta di un poema in sessanta ottave che si accompagna alle numerose sacre rappresentazioni dedicate al patrono fiorentino. La vicenda racconta la storia del Battista come riportata nel primo capitolo del Vangelo di Luca (Lc I, 5-25 e 39-80). La missione di Giovanni, definito "angelico trombetto», viene fatta iniziare a tre anni per il desiderio di penitenza che il bimbo nutre (il testo latino recita: "puer autem crescebat et confortabatur spiritu et erat in deserto usque in diem ostensionis suae ad Israhel》 Lc I, 80), (str. I6 e I 8):

[...] e in circa di tre anni sendo il fanciullo allevato et cresciuto, per fuggire e mondani dilecti e 'nganni quando poteva, non sendo veduto, soletto se n'andava nel giardino a contemplare il suo signor divino. 


\section{$[\ldots]$ e cominciò a ire verso il deserto essendo di cinqu'anni piccolino solecto ogni mattina et tutto il giorno stava et la sera faceva ritorno.}

Anche il cantico di Zaccaria è riportato, ridotto a un'ottava (str. 30), come forma di benedizione del padre al figlio per la sua missione. Segue quindi la narrazione, sempre derivata dal Vangelo di Luca (3, I-22), con la predicazione e il battesimo di Gesù. Non particolare attenzione è prestata alla danza che ne causa la morte, ridotta ad un'ottava, scelta che mostra l'intento devozionale ed educativo del testo.

Attingono abbondantemente alla materia biblica anche $i$ laudari, di grande diffusione a Firenze fin dal Trecento, come attesta la ricca tradizione manoscritta ad uso per lo più di confraternite. ${ }^{37}$ Non molte sono le edizioni a stampa dell'epoca poiché il laudario era un genere locale, circoscritto spesso a una sola confraternita. Per il periodo che ci interessa abbiamo un'edizione fiorentina delle laudi di diversi autori del I485-86, un volume di laudi di Feo Belcari del I490 circa, e una raccolta di laude vecchie e nuove edita intorno al I500. ${ }^{38}$ Queste pubblicazioni rappresentano una forma di canonizzazione del genere, una sorta di «raccolta aragonese» laudistica: come quella aveva codificato la lirica toscana, queste stabiliscono in qualche modo il canone del vasto materiale in circolazione, creando delle antologie sostenute da una precisa volontà celebrativa. ${ }^{39}$ In pieno Cinquecento la tradizione laudistica quattrocentesca viene riproposta a Firenze con l'edizione delle laude preparata dal domenicano, seguace di Savonarola, Serafino Razzi. ${ }^{\circ} \mathrm{Si}$ tratta di una pregevole antologia che raccoglie la produzione storica, dalle laude di Belcari a quelle di Lucrezia Tornabuoni, da quelle di Lorenzo de' Medici a quelle di Castellano Castellani, da quelle di Savonarola a quelle di Francesco degli Albizzi, con molte di diversi autori nemmeno noti (ma è inclusa come laude anche la canzone Vergine bella del Petrarca)..$^{4 \mathrm{I}}$

Trattandosi non di poesia narrativa, ma per musica, le fonti bibliche sono rintracciabili non in modo disteso, ma prevalentemente in singole parole, espressioni o figure. Come ha rilevato Concetto Del Popolo a proposito del laudario di San Gillio, le laude ricalcano «moduli liturgico-scritturali», per la loro composizione dunque «fanno da 
spunto, spesso, $i$ testi biblicis. ${ }^{42}$ Per esempio, la prima delle laude di questa confraternita è una riscrittura dell' annunciazione, un tema particolarmente caro alla laudistica fiorentina. Riporto qui alcune strofe del testo della lauda con a fianco la fonte biblica da cui dipende:

Benedecto sia el Sengnore, que' che Gabriel mandòe.

Ché 'l mandòe nella cittade, [《in civitatem» $L c \mathrm{I}, 26]$ in Naççareth d'oltremare, che dovesse salutare quella Vergine maggiore,

ch'a Gioseppo era sposata, per la legge congiugata, perciò li era accompagnata, ché di Dio fu servidore.

Intrando allei l'angelo sancto: [《ingressus ad eam» $L c \mathrm{I}, 28]$ «Ave», disse in quel, «cotanto [«Ave» $L c \mathrm{I}, 28]$ piena di Spirito sancto; benedecta sia tu, fiore».

Et la Donna salutata nella mente fu turbata; et pensava la beata lo saluto, con tremore.

[«turbata est in sermone eius» $L c \mathrm{I}, 29]$ [《et cogitabat qualis esset ista salutatio» $L c$ I, 29]

Allora disse Gabriello, quel clarito angelo bello: «Non temere, gilglio novello; ingratiata t’à il Segnore. [«Invenisti gratiam apud Deum» $L c$ I, 30]

Riceverai in ventre, beata, la Parola ch'ò parlata; quella fie di te incarnata; parturirai il Creatore».

Allora disse la beata:

«Come potrei essere infantata? 
Ch'io non seppi alcuna fiata che fosse homo». Per tal timore

l'angelo rispuose et disse: «Spirito sancto con largheççe, con virtute et con allegreççe fie di te obumbratore.

Lo sancto, che di te fie nato, Filgiuolo di Dio chiamato, per cui lo mondo fie salvato (poi serai sança dolore),

quel fie grande veramente, che fie Figliuol di Dio vivente, a la destra Dei sedente, sança fine regnatore.

Lo cui nome avrai a chiamare "Iesù" vero a salutare, quel fie nome principale del verace salvatore». ${ }^{43}$

Naturalmente la fonte biblica è elaborata e arricchita, ma la lauda resta un modo per veicolare la conoscenza biblica, di cui fruivano largamente gli illetterati, perfino i fanciulli. La lauda appare quindi come una forma di Biblia pauperum, una versione metrica delle Sacre Scritture, attraverso cui si educava alla fede anche l'analfabeta. Per esempio l'intero capitolo del sermone del monte è commentato in una lauda di Feo Belcari, Evangelio delle beatitudini esposto. ${ }^{44}$ Questi è considerato «il rappresentante maggiore di una forma espressiva che può essere additata a ragione come il vero motivo unificatore della vita culturale del tempos.45

A volte la Bibbia è rielaborata secondo una procedura più letteraria, cosi avviene per la salutatio angelica che Feo Belcari riscrive in laude da «cantasi come - I' veggio ben che 'l servir è vano». La lauda segue il modello della riscrittura in terzine del Padre nostro di Dante, facendo corrispondere a ogni espressione della salutatio angelica una terzina: 
Ave, del verbo eterno genitrice,

[Ave Maria]

Maria, de' peccator per pietà madre, grazie concedi a chi di te ben dice.

Piena di grazia ti fé 'l nostro padre, Signore e Dio del cielo e della terra, teco abitò per tue virtù leggiadre. Benedetta, finendo nostra guerra Tu se la porta di nostra salute, in tra le donne, vergin che non erra $E$ benedetto il re delle virtute Frutto acquistato di Spirito Santo. Del ventre tuo le grazie son venute. Gesù ci sciolse dall'eterno pianto, Santa Maria, per la tua umiltade, madre di Dio, ognun ti faccia canto. Priega il tuo Dio, fonte di pietade, per tutti i peccatori in questo punto, ed alla dimostrata umanitade, sicché ciascun sia per te assunto. ${ }^{46}$

Belcari prende a modello la Sacra Scrittura non solo perché ne riprende alcune forme, ma, come scrive Martelli, perché è

in quel testo, e solo in quel testo, che egli identifica l'unica manifestazione concessa alla conoscenza umana di autentica poesia. [...] le parole valgono, non in se stesse, ma per il significato che una lunga tradizione di esegesi scritturale ha depositato in ciascuna di esse. ${ }^{47}$

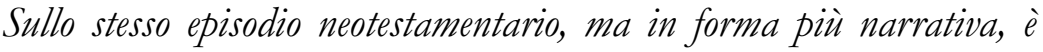
una lauda di anonimo, che presenta l'annunciazione dalla prospettiva della Vergine Maria, come se rispondesse alla domanda di un fedele interlocutore. Qui non viene volgarizzato il passo evangelico dell'annunciazione, ma presentata in forma narrativa l'iconografia dell'Annunciata (la Vergine che legge il profeta Isaia, il riferimento al giglio da parte dell'angelo). La vicenda è cosi attualizzata, come se fosse presente Maria, a cui il fedele domanda: "Dimmi, dolze Maria, a che pensavi / quando l'angiel t'apparse?», a cui Maria risponde:

Stavo nella camera mia soletta sopra la profezia, ch'è in Isaia, che dice la vergine conceperà 
e poi partorirà

[vocabit nomen eius Emmanuel» Is 7, I4]

Emanuel, che del ciel tien le chiavi.

Tacita stavo e nel pensier dicea:

$« \mathrm{O}$ dolce signor mio

concedi a me che la mortale iddea

che veder la poss'io: la virgin, che te Dio

eletta etternalmente ha a partorire,

ch'io la possa servire,

prima che le mie membra troppo aggravi».

Quest'era il pensier mio in tal dolcezza,

così mi stavo meco:

e quivi apparse una nuova chiareza

e il santo spirto seco, e disse «Dio è teco, [《Dominus tecum» Lc I, 28]

Tu se piena di grazia e benedetta

[《gratia plena» $L c \mathrm{I}, 28$ ]

tra tutte le donne eletta».

Temetti allor di que' parlar suavi.

L'angiol soggiunse e disse: «Non temere, [«Ne timeas» $L c$ I, 30$]$

o Maria graziosa,

i' son venuto per farti assapere,

che tu se' madre e sposa: candida, olente rosa,

partorirai dell'Altissimo il figlio, [《filius Altissimi» $L c$ I, 32 ]

a te conviensi il giglio

fatta se quella, a cui servir pregavi».

Intesi allor l'angelica favella,

rispuosi, con effetto:

«Ecco del mio Signore la sua ancella. [《Ecce ancilla Domini»»» Lc I, 38 ]

Sie fatto come ha detto».

[«fiat mihi secundum

Partissi il benedetto

verbum tuum»]

messo da Dio, e pien rimase il core [«discessit ab illa angelus» $L c$ I, 38 ]

di gaudio e di fervore

adempiuto quanto desideravi. ${ }^{48}$

L'annunciazione è un soggetto contemplato ovviamente anche nelle sacre rappresentazioni, in cui il genere lauda viene inglobato, come si vede nella Rappresentazione dell'annunziazione di nostra Donna con una aggiunta di dua capitoli bellissimi di Feo Belcari che andò in stampa a Firenze nel I495 circa. ${ }^{49}$ Si tratta di una pubblicazione di sole otto carte, ma l'opera è complessa e soprattutto solenne, le cui fonti sono, oltre alla Bibbia, anche san Bernardo e le Meditationes 
vitae Christi. ${ }^{\circ}$ Dopo il tradizionale annuncio della festa da parte dell'angelo (due ottave) seguitano le voci di trenta profeti (incluse le Sibille) introdotti dall'angelo, da Noè a Zaccaria, che annunciano il Messia, con un'ottava ciascuno. Le profezic occupano quasi cinque delle sei pagine in cui si svolge la festa (le altre due sono per il frontespizio, come si è detto, e per $i$ due capitoli, che però sono fuori dalla festa). Dopo Zaccaria compare Maria che prega Dio di poterlo servire e di conoscere la Vergine profetata. Infine si apre il cielo e Dio manda Gabriele ad annunciare, il quale è accompagnato da angeli che cantano una semplice lauda, che riprende il salmo I36:

Laudate el sommo Dio.

Laudatel con fervente e buon desio.

Laudando Dio cantando con buon zelo.

Laudate le virtù celeste et sante.

Laudate tutti quanti el Re del cielo.

Laudate le potentie tutte quante.

Dategli laude tante,

quante potete ad un Signor sì pio.

O lumi, o Sole, o Luna, o chiaro Sole

laudato sempre il giusto Dio eterno

che certo e' ci creò con sue parole.

Dunque laudate lui \& il suo governo

Laudinlo in sempiterno

che non dà mai e suo servi in oblio.

Laudate Dio, o Cieli \& suoi costumi

Laudate Dio, o fuoco o aria \& terra

\& voi venti \& larghi mari \& fiumi

laudate Dio che può dar pace \& guerra.

Laudando lui non si erra

perch'è gli è vero lume \& giusto Dio.

Perpetue laude gli dia tutto il mondo

le nube e venti, le bestie \& gli uccelli

gl'uomin, le donne, e pesci a tondo a tondo,

$\&$ tutte l'altre cose che fece elli

con dolci canti et belli

laudando Dio di gloria e con desio.

Laudate el sommo Dio

laudate con fervente e buon disio. ${ }^{51}$ 
Le laudi sono quasi dominanti in questo breve testo, infatti finita questa lauda un'altra è messa in bocca a Gabriele, che riassume il suo mandato e il significato dell'Incarnazione. Si direbbe una lauda educativa, che spiega il significato della festa:

Dal Cielo son mandato da Dio padre verace annuntiar la pace che perdonar vuol l'antico peccato ch'egli è Dì [Dio] innamorato dell'humana natura che gl'ha diliberato di pigliar carne pura di quella gentil figura la qual vi ho annuntiare che si debba preparare a ricever el figliuol di Dio beato. ${ }^{52}$

L'evento è raccontato poi con le parole evangeliche in latino: davanti a Maria Gabriele recita la salutatio completa. ${ }^{3}$ Seguono i due capitoli, uno rivolto a Maria Vergine, di dodici terzine, e uno agli angeli, pure di dodici terzine, in cui tutte le gerarchie angeliche sono invitate a lodare Dio. Anche questi capitoli sono in forma laudistica, infatti come «laude» viene definito nel testo il primo capitolo nell'invocazione alla Vergine: "ricevi in tuo onor la laude mia» (v. 3).

La diffusione della lauda e la sua costante pratica a Firenze, come attestata da molti documenti, crea dunque il terreno per una capillare conoscenza non passiva degli eventi della salvezza da parte del popolo. Al genere fu interessato anche Girolamo Savonarola, che, benché dichiarasse nell'Apologeticus il suo sospetto verso la poesia, che tradirebbe la semplicità delle Sacre Scritture, la praticò in gioventù e la usò poi anche in forma cantata. In questo scritto polemico diretto a Ugolino Verino, che rivendicava l'utilità della poesia cristiana, il frate replicava negando ogni utilità della poesia, in vista della finalità ultima dell'nomo, la salvezza eterna. Egli condannava anche la poesia cristiana:

E sebbene alcuni cattolici, condotti dallo zelo di Dio, per battere l'arroganza di alcuni vanitosissimi poeti che rimproveravano ai cristiani l'ignoranza dell'eloquenza, abbiano scritto in versi i Vangeli ed altre Scritture 
dell'Antico Testamento, tuttavia Dio non volle che fossero molto apprezzati nella sua Chiesa. Per cui non solo non si leggono, se non raramente, ma neppure sono molto considerati presso i cristiani, perché come l'eloquenza di questo secolo ha avuto inizio dai pagani così è piena della vanità dei (medesimi) pagani. ${ }^{54}$

Infine il domenicano consentiva ad approvare, ma di stretta misura, la bontà dell'intenzione educativa della poesia cristiana:

Concediamo che alcuni loro carmi conducano gli animi alla compunzione e richiamino i peccatori dal male; tuttavia ciò è cosa talmente rara che ai nostri tempi nessuno forse ha visto tale miracolo."s

Anche verso la musica sacra egli tenne un atteggiamento intransigente, condannando in varie prediche il modo di cantare laudistico, sia nei conventi che da parte di laici. Suggeriva di ricorrere piuttosto ai canti di Chiesa come Ave Maris Stella $e$ Veni Creator Spiritus. ${ }^{56}$ Tuttavia egli compose alcune laude (molte gli sono state attribuite, che però non sono sue). ${ }^{57}$ In particolare una di queste, la Laude al crocifisso, fu molto popolare e rimase a lungo nel patrimonio laudistico, anche dopo la morte del frate. Componimento giovanile, apparve a stampa nel 1492 allegata al Tractato dello amore di Iesù Christo, stampato a Firenze da Antonio Miscomini. La lauda si può considerare ovviamente biblica per il soggetto, che però è trattato con espressioni devote, che raggiungono talora un afflato mistico:

Iesù, sommo conforto,

Tu se tutto el mio amore

E'l mio beato porto

E santo redentore.

O gran bontà. Dolce pietà,

Felice quel che teco unito sta!

Oh quante volte offeso

T'ha l'alma e 'l cor meschino!

$\mathrm{E}$ tu sei in corce esteso,

per salvar me tapino.

O gran bontà, ecc.

$[\ldots]$ 
Iesù, fammi morire

Del tuo amor vivace;

Iesù, fammi languire

Con te, Segnor verace!

$\mathrm{O}$ gran bontà, ecc.

Iesù, foss'io confitto

Sopra quell'alto ligno,

Dove ti veggo afflitto,

Iesù, Segnor benigno!

O gran bontà, ecc. ${ }^{58}$

Questa lauda consta di dieci strofe bipartite, composte da una quartina di settenari con una ripresa di un doppio quinario seguito da un endecasillabo tronco. I ritornelli di coda hanno un evidente carattere a ballo, dunque è evidente l'intenzione di soppiantare la vana poesia profana. Nel codice Borromeo, che contiene le poesie di Savonarola scritte di suo pugno, sono abbinate al testo anche le notazioni musicali, di mano pure del frate. Ma si tratta non di una sua creazione, piuttosto di un contrafactum di altra lauda, Vergine tu mi fai, che discende dalla canzone profana Ançola tu mi fai. Il componimento godette in seguito di un'ottima tradizione musicale per tutto il Cinquecento. ${ }^{59}$

Delle laude Savonarola faceva un uso soprattutto educativo, evidentemente dovevano avere una funzione epuratrice rispetto alla musica da ballo, proprio per questo esse ricordano nella metrica e nella musica le canzoni profane. ${ }^{60}$ Questi interventi savonaroliani nella poesia laudistica bene si inquadrano nella prospettiva di un rinnovamento cristiano che intendeva fare di Firenze una città alleata a Dio, guida al bene in un mondo corrotto. La corruzione contemporanea non è solo oggetto delle sue prediche, ma anche delle sue prime poesie, che risalgono alla gioventù, ai tempi di Ferrara. Una condanna severa dei mali del mondo e della Chiesa del tempo si legge in Ruina mundi $e$ Ruina Ecclesiae, due canzoni che condannano i vizi del mondo: lussuria, superbia, ipocrisia, avarizia, e la trasformazione di Roma in «superba meretrice, Babilona». ${ }^{6 \mathrm{I}}$

Nella spinta verso la renovatio Savonarola assunse infine come esempio Mosè. Nelle prediche sopra l'Esodo colui che guida i figli d'Israele a fuggire dall'Egitto, ossia dalle tenebre del peccato, diventa il modello per il rinnovamento spirituale e politico della città di Firenze. Proprio a 
queste prediche, tenute allinizio della quaresima del I498, le ultime prima del processo e della condanna, aveva assistito Machiavelli, che ne relaziona in una lettera all'oratore fiorentino presso il papa, l'antisavonaroliano Ricciardo Becchi, datata 9 marzo dello stesso anno, pochi giorni dopo le prediche (tenute il 25 febbraio, 2 e 3 marzo). ${ }^{62}$ Machiavelli mostra nell'epistola, che ironizza sul frate pur riconoscendogli abilità politiche, di sovrapporre le qualità di Savonarola a Mosè, chiamando quest'ultimo «predicatore»:

L'altra mattina poi exponendo pure lo Exodo et venendo in quella parte, dove dice che Moyses amazò uno Egiptio, dixe che lo Egiptio erono gli huomini captivi, et Moyses el predicatore che gli amazava, scoprendo e vitii loro $\left[\ldots . .{ }^{63}\right.$

Se Mosè assume le caratteristiche del domenicano, si può dedurre ovviamente che il predicatore sia di rimando caratterizzabile come Mosè, ovvero che le due figure non siano disgiungibili per il Segretario fiorentino. Ma soprattutto si deve prendere atto che la conoscenza degli episodi di Esodo, che tanta parte hanno nella scrittura di Machiavelli, sia derivata dallinterpretazione del frate.

L'evoluzione di Firenze verso l'intransigenza e la radicale riforma di Savonarola risulta infine il coronamento di una lunga stagione di spiritualità e di studi religiosi fiorita in seno all'Umanesimo, di cui la passione per la Bibbia era il sostegno. Nonostante le critiche espresse dal Savonarola per le forme della poesia cristiana, ci si può chiedere se la condivisione delle narrazioni bibliche nelle pratiche legate alla sociabilità religiosa collettiva possa aver determinato tra $i$ Fiorentini una condizione favorevole per l'accoglienza di propositi radicali che riattualizzavano lo spirito che era stato quello del popolo di Israele. Si può forse supporre che sia stata Firenze, con il suo interesse per la Bibbia, di cui sono prova le molte riscritture bibliche, ad aver favorito la predicazione del domenicano perché corrispondeva a una disposiz̨ione già acquisita che trovava nello spirito profetico del frate la sua realizzazione.

Erminia Ardissino 
I. Nicolò Machiavelli, Il principe, a cura di Mario Martelli, Roma, Salerno 2006, pp. I I 3- I 23 е 3 I 2. Sui riferimenti biblici del Principe e in altre opere del Segretario fiorentino: John H. Geerken, Machiavelli Moses and Renaissance Politics, «Journal of the History of Ideas», 60 (I 999), pp. 579-595; Christopher Lynch, Macbiavelli on Reading the Bible Judiciously, «Hebraic Political Studies», I (2006), pp. I62-185; Marcia L. Colish, Republicanism, Religion, and Machiavelli's Savonarolan Moment, in Machiavelli and Religion: A Reppraisal, nella miscellanea su Machiavelli del «Journal of the History of Ideas», 6o (1999), pp. 597-616.

2. Nicolò Machiavelli, [Exortatione alla penitenza], in id., Tutte le opere, a cura di Mario Martelli, Firenze, Sansoni, I97 I, pp. 932-934: pp. 932-3.

3. Su questo aspetto si vedano: Sebastiano Gentile (a cura di), Umanesimo e Padri della Chiesa. Manoscritti e incunaboli di testi patristici da Francesco Petrarca al primo Cinquecento, Milano, Rose, I997; Dennis F. Lackner, The Camaldolese Academy: Ambrogio Traversari, Marsilio Ficino and the Christian Platonic Tradition, in Michael J. B., Allen and Valery Rees, with Martin Davies (a cura di), Marsilio Ficino: his Theology, his Philosophy, his Legacy, Leiden, Brill, pp. I4-44.

4. Sul vescovo: Luciano Cinelli e Maria Pia Paoli (a cura di), Antonino Pierozzi OP (I389-1459). La figura e l'opera di un santo arcivescovo nell'Europa del secolo XV (Firenze, 25-28 novembre 2009), Firenze, Nerbini, $20 \mathrm{I}_{3}$; Peter Francis Howard, Preaching and Theology in the Florence of Archbishop Antoninus I427-I459, Firenze, Olschki, I995.

5. Cito da Maria Pia Paoli, Antonino da Firenze O.P. e la direzione dei laici, in Gabriella Zarri (a cura di), Storia della direzione spirituale. III. L'età moderna, Brescia, Morcellania, 2008, pp. 85 -I 30.

6. I cinque poemetti, databili alla fine degli anni settanta, sono la Ystoria di Iudit e la Vita di Sancto Johanne Baptista (che si leggono in Fulvio Pezzarossa, I poemetti sacri di Lucrezia Tornabuoni, Firenze, Olschki, I974, pp. I 5 I-249), La istoria della casta Susanna (a cura di Paolo Orvieto, iconografia di Ornella Casazza, Bergamo, Moretti e Vitali Ed., I992), la Istoria di Ester e la Vita di Tubia (in Lucrezia Tornabuoni, Poemetti biblici, edizione critica a cura di Erminia Ardissino, Lugano, Ancora, 2016).

7. Giovanni Dominici, Lucula noctis, edizione a cura di Edmund Hunt, Notre Dame, University of Notre Dame Press, 1940. Su cui: Claudio Mésoniat, Poetica theologia. La Lucula noctis di Giovanni Dominici e le dispute letterarie tra '300 e '400, Roma, Edizioni di Storia e Letteratura, I984; N. Ben-Aryeh Debby, Renaissance Florence in the Rhetoric of Two Popular Preachers. Giovanni Dominici (1356-I419) and Bernardino da Siena (1380-I444), Turnhout, Brepols, 200I.

8. Su questa pagina della storia intellettuale fiorentina si possono vedere alcuni dei saggi che costituiscono il ricco capitolo L'età di Firenze (1378-1494), in Sergio Luzzato e Gabriele Pedullà (dir.), Atlante della letteratura italiana, Torino, Einaudi, 2010 , I, pp. 292-602; Paolo Viti, L'Umanesimo toscano del primo Quattrocento e Paolo Orvieto, L'Umanesimo toscano del secondo Quattrocento, in Enrico Malato (dir.), Storia della letteratura Italiana, Roma, Salerno, I994, pp. 2 I I-294 e pp. $295-405$. 
9. Per il commento delle epistole di san Paolo, che si trova alle pp. 425-472 dell'Opera omnia ficiniana (Basilea I 576 , r. an. Torino, Bottega d'Erasmo, i 963 ), si veda Cesare Vasoli, Le Praedicationes di Marsilio Ficino, in Ginetta Auzzas, Giovanni Baffetti, Carlo Delcorno (a cura di), Letteratura in forma di sermone: $i$ rapporti tra predicazione e letteratura nei secoli XIII-XVI Atti del seminario di studi, Bologna I 5-17 novembre 200I, Firenze, Olschki, 2003, pp. 9-28. Per il volgarizzamento dei salmi: Salterio abbreviato di s. Gerolamo (Breviarium Psalterii, in Patrologia Latina CXV, coll. I 45 I-6) si veda Paul Oscar Kristeller, Supplementum Ficinianum, Firenze, Olschki, I937, II, pp. I 8 5-7; Edoardo Barbieri, Panorama delle traduzioni bibliche in volgare prima del Concilio di Trento, Milano, CRELEB-CUSL Università Cattolica, 20 I I, pp. 39-40. In generale: $c f r$. Peter Serracino-Inglot, Ficino the Priest, in Marsilio Ficino: his Theology, his Philosophy, his Legacy, cit., pp. I-I 3.

ıo. Stefano Ugo Baldassarri (a cura di), Dignitas et excellentia hominis. Atti del Convegno internazionale di studi su Giannozzo Manetti. Georgetown University-Kent State University, Fiesole-Firenze, I 8-20 giugno 2007, Firenze, Le Lettere, 2008; Paul Botley, Latin Translation in the Renaissance. The Theory and Practice of Leonardo Bruni, Giannozzo Manetti and Desiderius Erasmus, Cambridge, Cambridge University Press, 2004; Annet den Haan, Giannozzo Manetti's New Testament: New Evidence on Sources, Translation Process and the Use of Valla's Annotationes, «Renaissance Studies», 28, (2014), pp. 73 I-747; John Monfasani, Criticism of Biblical Humanists in Quattrocento Italy, in Erika Rummel (ed.), Biblical Humanism and Scholasticism in the Age of Erasmus, Leiden, Brill, 2008, pp. I 5-38.

i . Si veda Francesco Bausi, Bibbia e Umanesimo, in Grazia Melli e Marialuigia Sipione (a cura di), La Bibbia e la letteratura italiana. Dal Medioevo al Rinascimento, Brescia, Morcellania, 2013, pp. 363-398: 370-383; Amos Hedelheit, Reading the Penitential Psalms in Late Fifteenth Century Florence, in Wim François e August Den Hollander (ed.) «Wading Lambs and Swimming Elephants». The Bible for the Laity and the Theologians in Late Medieval and Early Modern Era, Leuven-Paris, Peeters, 2012 , pp. 4I-54.

I 2. Su Sante Pagnini e gli studi biblici gravitanti intorno al Savonarola e a San Marco, dove il rabbino Blemet, che aveva preso i voti come domenicano nel I493, insegnava ebraico, si veda Timoteo Centi, L'attività letteraria di Sante Pagnini nel campo delle scienze bibliche, "Archivum Fratrum Praedicatorum», Is (1945), pp. 5-5 I.

I 3. Ancora inedito il poema del Verino, a cui si dedicò per due decadi, ma di cui ci sono rimasti solo alcuni libri. Cfr. Francesco Bausi, Ugolino Verino, Savonarola e la poesia religiosa tra Quattro e Cinquecento, in Studi savonaroliani. Verso il V centenario. Atti del Seminario di studi savonaroliani, Firenze, Accademia di Scienze e Lettere «La Colombaria», gennaio 1995, Firenze, SISMEL-Edizioni del Galluzzo, I996, pp. i27-i 35. Per il Theotocon: Domenico di Giovanni Da Corella, Theotocon, a cura di Lorenzo Amato, Roma, Edizioni di Storia e Letteratura, 2012.

I4. Guido Cortassi, Angelo Poliziano: «Padre nostro», in Luisa Secchi Tarugi (a cura di), Il sacro nel Rinascimento. Atti del XII convegno internazionale, ChiancianoPienza 17-20 luglio 2000, Firenze, Cesati, 2002, pp. 395-404; Maria Accame, L'interesse di Poliziano per gli autori cristiani. La traduzione di Atanasio, in Volgarizzare e 
tradurre dall'Umanesimo all'età contemporanea. Atti della giornata di studi 7 dicembre 20I I, Università di Roma La Sapienza, Tivoli, Tored, 20I3, pp. 47-8 I. Questa traduzione riguarda il commento ai Salmi.

I 5 . Simon Gilson, Dante and Renaissance Florence, Cambridge, Cambridge University Press, 2005; Alessandra Mita Ferraro, Matteo Palmieri. Una biografia intellettuale, Genova, Name, 2005.

I6. Sulla cultura teologica a Firenze si veda il ricco capitolo di Mario Martelli, Teologia e letteratura, in Id., Firenze, in Alberto Asor Rosa (dir.), Geografia e storia. II. L'età moderna, in Letteratura italiana, Torino, Einaudi, I988, pp. 104-167, che parla per Firenze di un vigoroso sentimento religioso che la critica illuministica e romantica consideravano superato dalla nuova temperie umanistica.

I7. Lorenzo DE’ Medici, Rime spirituali. La rapresentatione di San Giovanni e Paulo, a cura di Bernard Toscani, Roma, Edizioni di Storia e Letteratura, 2000. Cfr. Silvia Gazzano, Le laudi di Lucrezia Tornabuoni. Edirione critica, «Interpres», 32 (20 I 4), pp. I 52-230; Daniela Delcorno Branca, Lucrezia, Poliziano, Lorenzo, note su alcune convergenze fra lirica sacra e lirica profana, "Interpres», I9 (2000), pp. I I I-I 34; Maria Pia Sacchi, Lorenzo: poesia sacra e sacro nella poesia, in La Bibbia e la letteratura italiana, cit., pp. 455-470; Erminia Ardissino, Introduzione, in Tornabuoni, Poemetti biblici, cit., pp. I 3-5 I.

I 8. Francesco Caglioti, Donatello e i Medici. Storia del David e della Giuditta, Firenze, Olschki, 2000.

I9. Sull'importanza dell'ascolto di letture per la conoscenza biblica dei laici si veda Gordon Campbell, «Fides Ex Auditu»: Hearing and Reading the Bible in Emilia Ardissino e Elise Boillet (ed.), Lay Readings of the Bible in Early Modern Europe. Proceedings of the Conference Hold in Tours in 24-26 November 20Is, Leiden, Brill, in stampa.

20. Le informazioni derivano da Erminia Ardissino-Elise Boillet, Repertorio di letteratura biblica in Italiano a stampa (I463?-I650), Turnhoult, Brepols, in stampa.

2 I. Pseudo-Dante, Incomincia gli setti psalmi penitentiali unlgarmente composti. E prima domine ne in furore, Firenze, Johannes Petri, I $47 \mathrm{I}$. Circolava anche una versione in ottave di un anonimo, prima edizione Milano, I 493.

22. Rosanna Morace, I Salmi tra Riforma e Controriforma, «Studi (e testi) italiani», 35 (2015), pp. 55-8 I.

23. Sui salmi dello Pseudo-Dante vedi Ester Pietrobon, La penna interprete della cetra. I Salmi in volgare e la tradizione della poesia spirituale italiana nel Cinquecento, Tesi di Dottorato in Scienze linguistiche, Filologiche e Letterarie, Università di Padova, Ciclo XXVII, 201 5, pp. 21-34.

24. Per le traduzioni dei salmi di Benivieni e di Alamanni, cfr. Pietrobon, La penna interprete della cetra, cit., pp. 34-5 I; Morace, I Salmi tra Riforma e Controriforma, cit., pp. 58-67.

25. Guiliano Mambelli, Gli annali delle edizioni dantesche, Bologna, Zanichelli, I 93 I; D. E. Rhodes, The Early Editions of the Credo di Dante, «Cambridge Bibliographical 
Society Transaction», 9 (1 990), pp. 53 I-536; Massimo Seriacopi. Il Credo di Dante, il Compendio della Comedia di Cecco degli Ugurgieri e il Capitolo di Jacopo Alighieri: qualche notazione, in Id., Intorno a Dante, Firenze, Libreria Chiari, 2004, pp. 49-82.

26. I temi coprono sia il Vecchio sia il Nuovo Testamento, si accentrano sulle figure emblematiche, come la Vergine (molte sono le feste dell'Annunciazione), il Battista, Abramo, Isacco, Giacobbe, Giuseppe, Ester, Tobia, Giobbe, Mosé, il figliuol prodigo, Lazzaro, Marta. Per un'essenziale bibliografia cfr: Sacre rappresentaz̧ioni fiorentine del Quattrocento, a cura di Giovanni Ponte, Milano, Marzorati, I974; Sacre rappresentazioni del Quattrocento, a cura di Luigi Banfi, Torino, UTET, I963; Nuovo corpus di sacre rappresentazioni fiorentine del Quattrocento, a cura di Nerida Newbigin, Bologna, Commissione per i Testi di Lingua, I983. Per gli studi più recenti: Paola Ventrone, Lo spettacolo religioso a Firenze nel Quattrocento, Milano, Università cattolica del S. Cuore, 2008; Pietro Delcorno, Lazzaro e il ricco Epulone. Metamorfosi di una parabola fra Quattro e Cinquecento, Bologna, Il mulino, 20 I 4; Paola Ventrone, Teatro civile e sacra rappresentazione a Firenze nel Rinascimento, Firenze, Le lettere, 20I6; Nerida Newbegin, The World Made Flesh. The Rappresentazioni of Mysteries and Miracles in Fifteenth-Century Florence, in Timothy Verdon e John Henderson (ed.), Christianity and the Renaissance. Image and Religious Imagination in the Quattrocento, Syracuse, Syracuse University Press, pp. 362-368.

27. La poesia religiosa: $i$ cantari agiografici e le rime di argomento sacro, a cura di Alfredo Cioni, Firenze, Sansoni, 1963. Sui canterini in genere Michelangelo Picone e Maria Bendinelli Predelli (a cura di), I cantari. Struttura e tradizione. Atti del convegno di Montréal I9-20 marzo ı 98 I, Firenze, Olschki, I984; Michelangelo Picone e Luisa Rubini (a cura di), Il cantare italiano fra folklore e letteratura, Firenze, Olschki, 2007; Luca Degl'Innocenti, «Al suon di questa cetra». Ricerche sulla poesia orale del Rinascimento, Firenze, Società Editrice Fiorentina, 2016. Manca però uno studio specifico sui cantari di argomento sacro.

28. Cfr. Lirici toscani del Quattrocento, a cura di Antonio Lanza, Roma, Bulzoni, 1973-1975, p. 169.

29. Cfr. La Istoria di Susanna e Daniello, poemetto popolare italiano antico, a cura di A. Parducci, «Romania», XLII (191 3), I65, pp. 34-75; Irene Tani, Niccolò Cieco, in Dizionario biografico degli Italiani, Roma, Istituto Italiano per l'Enciclopedia, 2013 , vol. 78 , p. 387 .

30. Niccolò Cicerchia, La passione di Gesù Cristo, Firenze, S. Jacopo a Ripoli, [c. I482]. Si veda Cantari religiosi senesi del Trecento. Neri Pagliaresi, fra Felice Tancredi da Massa, Niccolò Cicerchia, a cura di Giorgio Varanini, Bari, Laterza, i 965 . Un terzo poema del Cicerchia, La vendetta di Cristo, in realtà non è biblico, ma tratta di un miracolo avvenuto ai tempi di Vespasiano.

3 I. L'edizione critica delle rime di Bernardo Pulci a cura di Fabio Barracalla è in corso di stampa. La recente voce di Alessio Decaria, Pulci, Bernardo, in Dizionario Biografico degli Italiani, 2016 , vol. 85 , pp. 660-662, gli attribuisce la Passione di Cristo e il Lamento di Maria Maddalena, che esce però solo nel 'soo. Ho visto l'edizione: Bernardo Pulci, La passione del nostro Signore Gesù Cristo, Poemetti sacri, Firenze, Francesco Bonaccorsi, 3.XI.1490. 
32. Pulci, La passione, c. a2r. Sulle suore Murate cfr. Kate J.P. Lowe, Female Strategies for Success in a Male-Ordered World. The Benedictine Convent of Le Murate in the Fifteenth and Early Sixteenth Centuries, "Studies in Church History», 27 (1990), pp. 209-2 I; Giuseppe Zippel, Le monache d'Annalena e il Savonarola, «Rivista d'Italia», 3 (I $90 \mathrm{I})$, pp. 23 I-49. Le trascrizioni da antiche stampe rispettano la forma, si interviene solo a distinguere la $u$ da $v$, inserendo i segni diacritici e la punteggiatura secondo gli usi moderni.

33. Pulci, La passione, c. a4r, ottava i. Cfr. l'incipit del sonetto rinterzato di Dante: «O voi che per la via d'Amor passate / attendete e guardate / s'elli è dolore alcun, quanto 'l mio, grave» in DANTE, Vita nova, 2, I4; all'ottava quinta si legge ad esempio: «che prese l'huom per trapassar del segno», che è ripresa di Paradiso XXVI, i 17 .

34. Benedetto Pulci, Iterum in passione Domini, in Pulci, La passione.

35. Pulci, Il pianto della Maddalena, in ID., La passione.

36. [Firenze, Lorenzo Morgiani, c. I495]. Altre storie sul Battista: Vita di San Giovanni Battista, [Firenze, Lorenzo Morgiani, c. I 496-I 497]; Questa e la nita et morte di San Gionanni [!] Baptista, [Firenze, s.i.t., dopo il I 500].

37. Cfr. Gilberto Aranci (a cura di), Il laudario fiorentino del Trecento, Montespertoli, Aleph, 2002; Laude fiorentine. Il laudario della compagnia di san Gillio, a cura di Concetto del Popolo, Firenze, Olschki, I990; Renzo Rabboni, Laudari e canzonieri nella Firenze del '400. Scrittura privata e modelli nel Vat. Barb. Lat. 3679, Bologna, CLUEB, I991, che si occupa di questo ricco codice. Cfr. Giulio Cattin, Sulla evoluzione musicale e letteraria della lauda nei secoli XIV e XV, «Quadrivium», 2 (1958), pp. 44-75; Id., La lauda, in Id., Il Quattrocento, in Alberto Asor Rosa (dir.), Letteratura italiana. VI. Teatro, musica, tradizione dei classici, Torino, Einaudi, 1986, pp. 3 I $3-8$.

38. Feo Belcari, Laude, [Firenze, Stampatore del Virgilio, circai 49o]; di più autori, incluso Feo Belcari, a cura di Jacopo de' Morsi Laude di Dio, Firenze, Francesco Bonaccorsi, I485/86 e [Firenze, Bartolomeo di Libri, circa 1495]; Laude vecchie e nuove, [Firenze, Bartolomeo di Libri], per Piero Pacini, [circa i 500 ]. Feo Belcari è attivo già a metà del secolo: nato nel i $4 \mathrm{I}$ o morì nel I 484 , la fortuna delle sue laudi fu valorizzata dalla stampa.

39. Cfr. Rabboni, Laudari e canzonieri nella Firenze del '400, cit., p. 75. Ancora nel Seicento Firenze resta fedele a questa sua tradizione, ne è un esempio la raccolta di laude Rime sacre edite nel i680. Sui laudari fiorentini Cyrilla Barr, The Monophonic Lauda and the Lay Religious Confraternities of Tuscany and Umbria in the Late Middle Ages, Kalamazoo, Medieval Institute Publications Western Michigan University, I 988; Blake Wilson, Music and Merchants. The Laudesi Companies of Republican Florence, Oxford, Clarendon Press, I992; Id., Singing Poetry in Renaissance Florence. The cantasi come Tradition (1375-I550), Firenze, Olschki, 2009.

40. Cfr. Anne Piéjus, Musique et dévotion à Rome à la fin de la Renaissance: les laudes de l'Oratoire, Turhout, Brepols, 2013. 
4I. Libro primo delle laudi spirituali da diversi eccell. e divoti autori, antichi e moderni composte, ... raccolte dal $R$. P. Fra Serafino Razzi, Venezia, ad instanzia dei Giunti di Firenze, 1563.

42. Laude fiorentine, p. 77.

43. Ibid., pp. ios-i Io, le note a p. 77.

44. Laude spirituali di Feo Belcari, Firenze, Molini e Cecchi, I863, pp. 6-7.

45. Rabboni, Laudari e canzonieri nella Firenze del '400, cit., p. $7 \mathrm{I}$.

46. Laude spirituali di Feo Belcari, p. 4. Si può notare come questa riscrittura contenga anche la parte oltre la salutatio angelica, che era stata aggiunta solo in quel secolo dal francescano Marco dal Monte Santa Maria (cfr. Marco dal Monte Santa Maria, Tabula de la salute, Venice, Nicolaus Belaguer, I496).

47. Martelli, Firenze, cit., pp. i I 4-i I s.

48. Laude spirituali di Feo Belcari, cit., p. 87.

49. Feo Belcari, La rappresentazione dell'annunziazione di nostra Donna, [Firenze, Bartolomeo de' Libri, circa I 495].

50. Cfr. Martelli, Firenze, cit., p. I I 8.

$5 \mathrm{I}$. Feo Belcari, La rappresentazione dell'annunziazione di nostra Donna, in ID., Sacre rappresentazioni e laude, a cura di Onorato Allocco-Castellino, Torino, UTET, pp. 53-76: 72-3.

52. Ibid., p. 73.

53. «Ave Maria, gratia plena, Dominus tecum, benedicta tu in mulieribus. $\mathrm{Ne}$ timeas, Maria, invenisti enim gratiam apud Deum, ecce concipies in utero et paries filium, et erit nomen eius Iesus. Hic erit magnus et Filius Altissimi vocabitur, et dabit illi Dominus Deus sedem David patris eius, et regnabit in domo Jacob in eternum, et Regni eius non erit finis. / La Vergine Maria risponde all'angelo Gabriello / Quomodo fiet istud, quoniam virum non cognosco? / L'angiol Gabbriello risponde: / Spiritus Sanctus superveniet in te, et virtus Altissimi obumbrabit tibi: ideoque et quod nascetur ex te sanctum, vocabitur Filius Dei, et ecce Elisabeth, cognata tua, et ipsa concepit filium in senectute sua; et hic mensis sextus est illi, quae vocatur sterilis, quia non erit impossibile apud Deum omne verbum. / La Vergine Maria risponde: Ecce ancilla Domini; fiat mihi secundum verbum tuum», ibid., pp. 73-74. Cfr. Lc I, 28-33.

54. Girolamo Savonarola, Apologetico. Indole e natura dell'arte poetica, a cura di A. Stagnitta, Roma, Armando, I 998 , p. 90.

55. Ibid., p. го3.

56. Cfr. Patrick Macey, Bonfire Songs. Savonarola's Musical Legacy, Oxford, Clarendon Press, I998, pp. 9I-8. 
57. Cfr. ibid., pp. 98-100.

58. Girolamo Savonarola, Rime, a cura di Giona Tuccini, Genova, Il melangolo, 20I 5, pp. 173-4. Cfr. Mario Martelli, Savonarola poeta, in Gian Carlo Garfagnini (a cura di), Una città e il suo profeta. Firenze di fronte al Savonarola, Firenze, Sismel, 200I, pp. I 27-I 37. Sull'esegesi savonaroliana delle Scritture cfr. il sintetico ma ricco saggio di Salvatore Camporeale, Savonarola lettore e commentatore del testo sacro, nella raccolta appena menzionata a pp. I 83-200.

59. Savonarola, Rime, p. 175-176. Per i vari compositori che diedero la musica alla lauda, cfr. Macey, Bonfire Songs. Savonarola's Musical Legacy, cit., pp. Iо2-104; Francesco Luisi, «Cantasi a ballo». Annotazioni formali su alcune laude savonaroliane, in Una città e il suo profeta, cit., pp. 435-449: 436.

6o. Cfr. Blake Wilson, «Hora mai sono in età»: Savonarola and Music in Laurential Florence, in Una città e il suo profeta, cit., pp. 283-310.

6i. Savonarola, Rime, cit., p. 96. Sull'influenza di Petrarca sul Savonarola poeta cfr. Rossella Bessi, Girolamo Savonarola petrarchista (e una nota sul primo soggiorno fiorentino), in Studi savonaroliani verso il V centenario, cit., pp. I 37-1 47.

62. Machiavelli, Lettere, pp. 910-2. Sulla lettera: Mario Martelli, Savonarola e Machiavelli, in Gian Carlo Garfagnini (a cura di), Savonarola, democrazia, tirannide, profezia, Firenze, SISMEL, I998, pp. I67-90; Cfr. Innocenzo Cervelli, Savonarola, Machiavelli e il libro dell'Esodo, ibid., pp. 243-298; Claudio Leonardi, Savonarola e la politica nelle prediche sopra l'Esodo e nel "Trattato circa el reggimento e governo della città di Firenze», in Gian Carlo Garfagnini (a cura di), Savonarola e la politica. Atti del Seminario (Firenze, 19-20 ottobre 1996), Firenze, SISMEL - Edizioni del Galluzzo, I997, pp. 75-89.

63. Machiavelli, Lettere, cit., pp. 9i r. Rileva il lapsus e il suo significato Cervelli, Savonarola, Machiavelli e il libro dell'Esodo, cit., pp. 284-5. 